\title{
Étude expérimentale du développement larvaire d'Hymenolepis stylosa (Rudolphi, 1809) Raillet, 1899
}

\author{
(Cestode, Cyclophyllide)
}

\author{
par C. GABRION \\ Laboratoire de Parasitologie comparée, \\ U.S.T.L., Place E.-Bataillon, F 34060 Montpellier Cedex.
}

\section{Résumé.}

L'étude comparée du développement larvaire d'Hymenolepis stylosa (Rudolphi, 1809) (Cestode, Cyclophyllide), parasite de Corvidés, a été réalisée expérimentalement chez trois Insectes.

L'observation du développement chez un Coléoptère, Tenebrio molitor, montre que le scolex se différencie avant l'invagination du métacestode dans la vésicule cystique, et que le cysticercoïde est pourvu d'un cercomère long et flexueux. Par sa morphologie et son évolution, le cysticercoïde d' $H$. stylosa est semblable aux cysticercoïdes des autres Hymenolepis d'Oiseaux dont le développement est connu.

Le développement chez un Orthoptère, Locusta migratoria, est identique à celui observé chez T. molitor, mais l'invagination du scolex intervient plus rapidement.

Chez un autre Coléoptère, Dermestes frischi, l'oncosphère est bloquée au niveau de la paroi de l'intestin.

Il résulte de ces observations qu'une étude comparée de la structure des cysticercoïdes d'Hymenolepis de Mammifères et d'Oiseaux s'impose pour rechercher des affinités entre les espèces appartenant à ce genre.

\section{Summary.}

Experimental study of the larval development of Hymenolepis stylosa (Rudolphi, 1809), Raillet, 1899 (Cestoda : Cyclophyllidea).

Comparative studies of the larval development of Hymenolepis stylosa Rudolphi, 1809 (Cestoda: Cyclophyllidea), a parasite of Corvid birds are undertaken from three Insect species.

Accepté le 21 décembre 1976. 
The development in the beetle, Tenebrio molitor shows that the scolex differenciation occurs before the invagination of the metacestode in the cystic vesicle. The cercomer is long, narrow and flexuous.

In the grasshopper, Locusta migratoria, the development is the same one but the scolex invagination begins early.

In another beetle, Dermestes frischi, the oncosphere is stopped in the gut-wall.

The morphology and development of the cysticercoids of avian species of Hymenolepis, which have a well known life cycle, are similar.

Studies on the structure of the larval stages of avian and mammal species of Hymenolepis seem necessary to find the relations between the different species of this genus.

Le genre Hymenolepis Weiland, 1858, compte un très grand nombre d'espèces parasites d'Oiseaux, mais aussi de Mammifères (Rongeurs, Chiroptères et Insectivores). Les larves cysticercoïdes sont hébergées par des Invertébrés aquatiques: Crustacés, Oligochètes ou Mollusques, mais aussi par des Invertébrés continentaux appartenant à tous les groupes, à l'exception des Isopodes.

Il nous a paru intéressant de rechercher si la nature du milieu dans lequel se déroule le cycle et si l'éclectisme observé dans le choix de l'hôte intermédiaire et définitif pouvaient avoir des répercussions sur la morphologie et l'évolution des formes larvaires dans l'hôte intermédiaire.

Cependant. si nous possédons des données sur le développement des Hymenolepis d'Oiseaux aquatiques d'une part, et de ceux parasites de Rongeurs et d'Insectivores d'autre part, nos connaissances sur celui des Hymenolepis d'Oiseaux continentaux, représentés essentiellement par les Passeriformes, est très imparfaite.

Dans ce travail, nous nous sommes attaché à suivre expérimentalement le développement larvaire d'un Cestode de Corvidés, Hymenolepis stylosa Rudolphi, 1809, chez un Coléoptère, Tenebrio molitor, et à établir le mode d'évolution du cysticercoïde. Enfin, nous avons essayé de réaliser le passage de l'oncosphère dans deux autres Insectes: un Coléoptère, Dermestes frischi, et un Orthoptère, Locusta migratoria.

\section{Matériel et méthodes}

Les adultes d'H. stylosa sont récoltés dans l'intestin moyen de Pies, Pica pica, et de Choucas, Coloeus monedula, capturés dans la région de Montpellier.

L'infestation des Tenebrio adultes $\delta$ et $q$ est réalisée en mêlant les œufs recueillis dans les segments gravides des Cestodes à la nourriture des Coléoptères, à jeun depuis 48 heures. Celle des larves de Dermestes se fait à partir d'anneaux mûrs ajoutés à la nourriture. Enfin, pour les Orthoptères, les segments gravides sont placés directement entre les pièces buccales. Dans tous les cas, les animaux infestés sont maintenus, durant toute la durée de l'expérimentation, à une température constante $\left(28^{\circ} \mathrm{C}\right.$ pour les Coléoptères, $34^{\circ} \mathrm{C}$ pour les Orthoptères). 
Les Insectes sont sacrifiés toutes les 24 heures et les cysticercoïdes recherchés dans la cavité abdominale.

L'infestation expérimentale des Oiseaux est réalisée en faisant ingérer à des Choucas des Insectes chez lesquels l'importance de l'infestation par les cysticercoïdes a été déterminée par une rapide dissection de contrôle.

Pour établir la table chronologique du développement, nous avons utilisé, pour les différents stades, la terminologie établie par Voge et Heyneman (1957) pour $H$. diminuta et $H$. nana.

\section{Résultats}

\section{Cestode adulte.}

Bien que ce Cestode soit largement répandu chez la plupart des Corvidés d'Europe, les descriptions données par les divers auteurs restent imprécises. La grande ressemblance d' $H$. stylosa avec un autre Hymenolepis de Passeriforme, $H$. fringillarum, ajoute à la confusion. Mettrick (1968), dans sa revue des Helminthes parasites des Oiseaux de l'Hertfordshire, insiste sur la difficulté qu'il y a à séparer les deux espèces.

H. stylosa est un Cestode de $100 \mathrm{~mm}$ environ, fixé à la muqueuse intestinale, entre l'anse duodénale et les caecums digestifs.

Le scolex, de 250 à $280 \mu \mathrm{m}$, présente 4 ventouses inermes de 80 à $100 \mu \mathrm{m}$ de diamètre et un rostre armé d'une rangée de 10 crochets, longs de 28 à $35 \mu \mathrm{m}$ (fig. 7 , Pl. II b).

Le pore génital s'ouvre toujours à droite, au tiers supérieur des segments.

L'appareil génital comprend trois testicules (120-150 $\mu \mathrm{m}$ de diamètre), deux antiporaux et un poral, disposés en triangle. La poche du cirre, globuleuse, mesure 200 à $250 \mu \mathrm{m}$ de long sur 80 à $140 \mu \mathrm{m}$ de diamètre. La vésicule séminale antérieure est très développée. L'ovaire, bilobé, est situé ventralement par rapport au testicule antiporal postérieur. La glande vitelline est compacte, située entre les deux testicules postérieurs. L'utérus médian, bilobé au début, devient sacciforme.

\section{Les œufs (fig. 1).}

Les œufs observés dans une solution physiologique mesurent $98 \times 88 \mu \mathrm{m}$. A l'intérieur, l'oncosphère, limitée par un mince embryophore, ne mesure que $45 \times 48 \mu \mathrm{m}$. Elle est armée de trois paires de crochets, longs de $22 \mu \mathrm{m}$. Les crochets de chaque paire latérale, reliés à leur base, fonctionnent de manière synchrone. Les crochets médians, indépendants l'un de l'autre, sont plus minces que les latéraux. L'espace compris entre l'embryophore et la coque de l'œuf est occupé par l'enveloppe vitelline, granuleuse. 


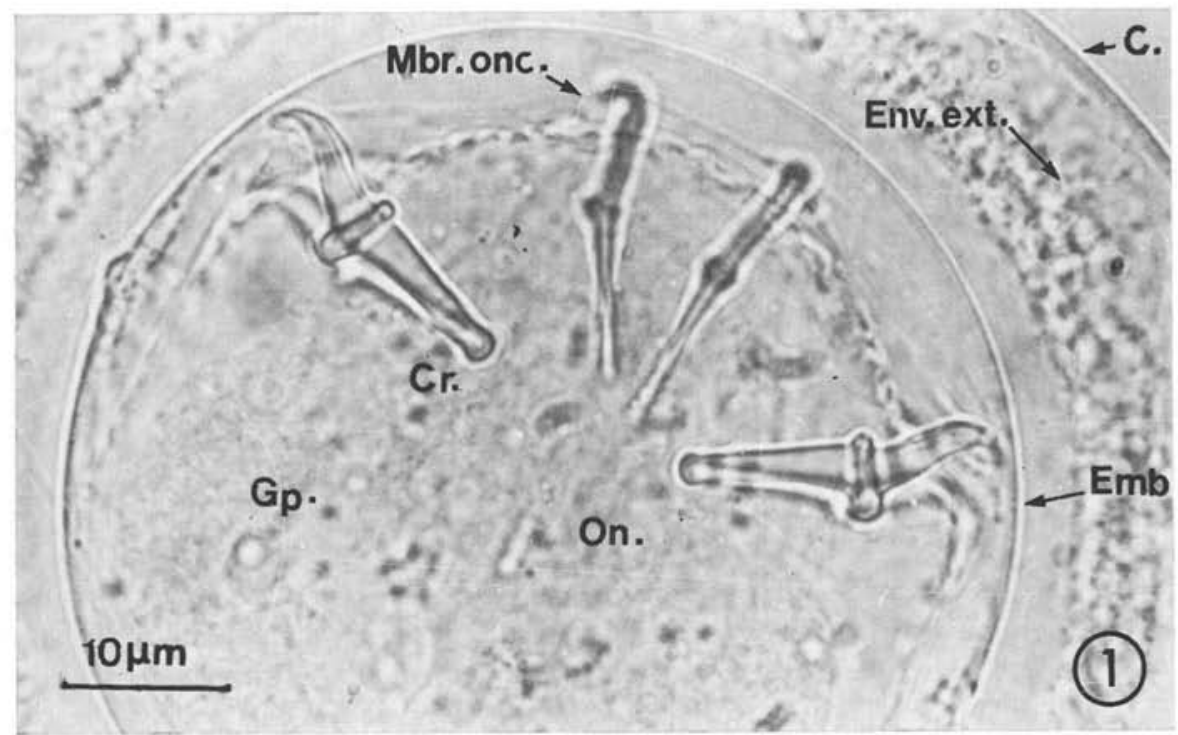

Fig. 1. - H. stylosa. Oncosphère mûre mettant en évidence les différentes enveloppes. C. Coque de l'œuf; Cr. Crochet; Emb. Embryophore; Env. ext. Enveloppe externe ; G.P. Glande de pénétration; Mbr. onc. Membrane oncosphérale; On. Oncosphère.

\section{Localisation des stades larvaires.}

L'oncosphère, libérée dans le tube digestif de l'Insecte, pénètre activement au niveau du mésentéron, en avant des tubes de Malpighi. Traversant la paroi intestinale, elle se place entre les cellules adipeuses qui tapissent le tube digestif.

Les cysticercoïdes sont très proches les uns des autres, et leurs longs cercomères, plus ou moins enchevêtrés, les «amarrent» au tube digestif ou aux expansions des tubes de Malpighi.

Dans le cas d'hyperinfestation, nous avons observé, surtout chez les Orthoptères, la présence de cysticercoïdes dans la musculature pleurale et contre les organes génitaux; la localisation la plus habituelle reste néanmoins la paroi du mésentéron.

\section{Evolution de la larve chez Tenebrio molitor.}

Nos observations concernent exclusivement les stades larvaires II, III et IV obtenus chez les Tenebrio $\sigma^{t}$ et $q$.

a) Embryon de 5 à 6 jours (Stade II) (fig. 2, Pl. I a).

Cinq jours après l'infestation, les larves observées dans la cavité générale de l'Insecte n'ont plus la forme sphérique qui caractérise l'hexacanthe libre. Elles sont 
ovoïdes et mesurent 360 à $600 \mu \mathrm{m}$ de longueur et 300 à $400 \mu \cdot \mathrm{m}$ de largeur, et présentent, à la partie postérieure, un cercomère long de 120 à $260 \mu \mathrm{m}$. La vésicule cystique est bien développée.

Au $6^{\circ}$ jour, une intense prolifération cellulaire, dans la paroi de la vésicule cystique, annonce l'apparition du futur scolex (fig. 2), à l'opposé du cercomère dans lequel s'effectue la migration des crochets de l'oncosphère.

b) Embryon de 7 à 8 jours (Stade III) (fig. 3, Pl. I b et c).

Entre le $7^{\circ}$ et le $8^{\circ}$ jour, la division de la larve en trois parties est achevée. La région antérieure, le métacestode, mesure $310 \mu \mathrm{m}$ de longueur et $140 \mu \mathrm{m}$ de largeur. Il présente quatre ventouses et un rostre sur lequel apparaissent des ébauches des crochets (fig. 3).

La partie moyenne, sphérique (400 u.m de diamètre), renferme la vésicule cystique. Dans la paroi se différencient les fibres musculaires. La cavité centrale est limitée par une couche fibreuse.

Le cercomère, allongé, mesure $800 \mu \mathrm{m}$. Deux paires de crochets de la larve hexacanthe, parfois trois, se retrouvent à son extrémité.

Le système excréteur est constitué par un anneau circumrostral d'où partent quatre canaux excréteurs. Les deux dorsaux reçoivent les canaux secondaires provenant des protonéphridies. Les deux ventraux, plus gros, viennent déboucher dans la partie postérieure du cysticercoïde, de part et d'autre du cercomère. Il ne semble pas y avoir de vessie excrétrice.

c) Embryon de 8 à 9 jours (Stade IV).

Entre le $8^{\circ}$ et le $9^{\circ}$ jour, se produit l'invagination du métacestode, qui entraîne la réduction de la vésicule cystique.

Le cysticercoïde, enveloppé d'un glycocalyx abondant, mesure à présent entre 370 et $450 \mu \mathrm{m}$ de longueur et 230 à $350 \mu \mathrm{m}$ de largeur.

A l'intérieur de la cavité d'invagination, le métacestode est mobile; il présente un scolex bien différencié, pourvu de 4 ventouses et d'un rostre, armé de 10 crochets. Ceux-ci n'ont pas encore acquis leur taille définitive et mesurent entre 25 et $28 \mu \mathrm{m}$.

Les corpuscules calcaires sont disposés en manchon autour du métacestode (fig. 4, Pl. I d).

Le cercomère, long et flexueux, est boursouflé par endroit ; il continue à s'allonger et mesure plus de $500 \mu \mathrm{m}$ (fig. 3).

d) Stade IV âgé (fig. 4 et 5, Pl. I d; fig. 6, Pl. II).

14 jours (fig. 4, Pl. I d) après le repas infestant, la taille du cysticercoïde n'a pas varié. Nous n'avons pas observé de réduction de taille consécutive à l'invagination du métacestode, comme l'avait observé Schiller (1959) chez H. nana. On observe toujours des individus dont la taille varie entre 380 et $450 \mu \mathrm{m}$ de longueur et 230 à $350 \mu \mathrm{m}$ 
de largeur. Les plus petits sont souvent sphériques, alors que les grands individus sont nettement ovoïdes.

Le scolex (fig. 6, Pl. II a) du métacestode mesure $400 \mu \mathrm{m}$ de diamètre; il est pourvu de 4 ventouses de 82 à $90 \mu \mathrm{m}$ de longueur et 60 à $70 \mu \mathrm{m}$ de largeur. Le rostre mesure $130 \times 60 \mu \mathrm{m}$ et porte une couronne de 10 crochets (fig. $7, \mathrm{Pl}$. II b) de $32 \mu \mathrm{m}$ de longueur, identiques à ceux de l'adulte.

Le cercomère continue de s'allonger et mesure plus de $1 \mathrm{~mm}$. Il est plus large et plus court chez les individus sphériques de petite taille.

Les crochets de l'hexacanthe sont dispersés dans le parenchyme du cercomère. Certains ont une lame ébréchée, d'autres présentent des signes de dégénérescence mélanique.

A 26 jours (fig. 5), les cysticercoïdes sont infestants.

37 jours après, ils sont toujours actifs à l'intérieur des Tenebrio. Le cercomère mesure à présent plus de $3 \mathrm{~mm}$.

e) Infestation de l'hôte définitif.

Le passage de l'Insecte à l'Oiseau a été réalisé chez Coloeus monedula. Des Tenebrio infestés depuis 26 jours ont été donnés à un Choucas. Celui-ci, 15 jours après ingestion des Insectes parasités, présentait, à la dissection, $70 \mathrm{H}$. stylosa. Les Cestodes étaient localisés à la partie terminale du tube digestif, sur un étroit segment $(16 \mathrm{~cm})$. Tous les Cestodes étaient au même stade du développement; aucun segment ne contenait d'œufs.

\section{Etude du développement chez Locusta migratoria.}

Le développement d' $H$. stylosa chez $L$. migratoria ne diffère pas fondamentalement de celui décrit chez $T$. molitor. Néanmoins, nous avons observé que l'invagination du métacestode intervient dès le $7^{\circ}$ jour, alors qu'elle ne se produit, chez $T$. molitor, qu'entre le $8^{\circ}$ et le $9^{\circ}$ jour. Cette accélération, en accord avec les observations effectuées par de nombreux auteurs chez d'autres Cestodes (Freeman, 1952; Heyneman,

Fig. 2 à 5. - H. stylosa. Différentes phases du développement.

FIG. 2. - Cysticercoïde à 5-6 jours (Stade II). Le cercomère (Cer.) est bien développé, alors que 'e scolex n'apparaît que sous forme d'un massif cellulaire (Mas. cel.); Vés. cys. Vésicule cystique ; Cr. Crochet.

Fig. 3. - Cysticercoïde à 8 jours (Stade III). Le cysticercoïde est formé de trois parties bien distinctes: le scolex (Sc.), bien différencié, est muni d'un rostre (R.) armé et de 4 ventouses (V.); la vésicule cystique (Vés. cys.) et le cercomère (Cer.).

FIG. 4. - Cysticercoïde à 14 jours (Stade IV). Le scolex (Sc. inv.) est invaginé à l'intérieur de la vésicule cystique. Les crochets rostraux sont bien développés. Les corpuscules calcaires (C.c.) entourent le métacestode. Fb. Fibres.

FIG. 5. - Cysticercoïde à 24 jours (Stade IV âgé). Le cysticercoïde n'est pas modifié. Seul le cercomère (Cer.) a subi un allongement. Il mesure plus de $4 \mathrm{~mm}$. 


$$
\text { Q }
$$




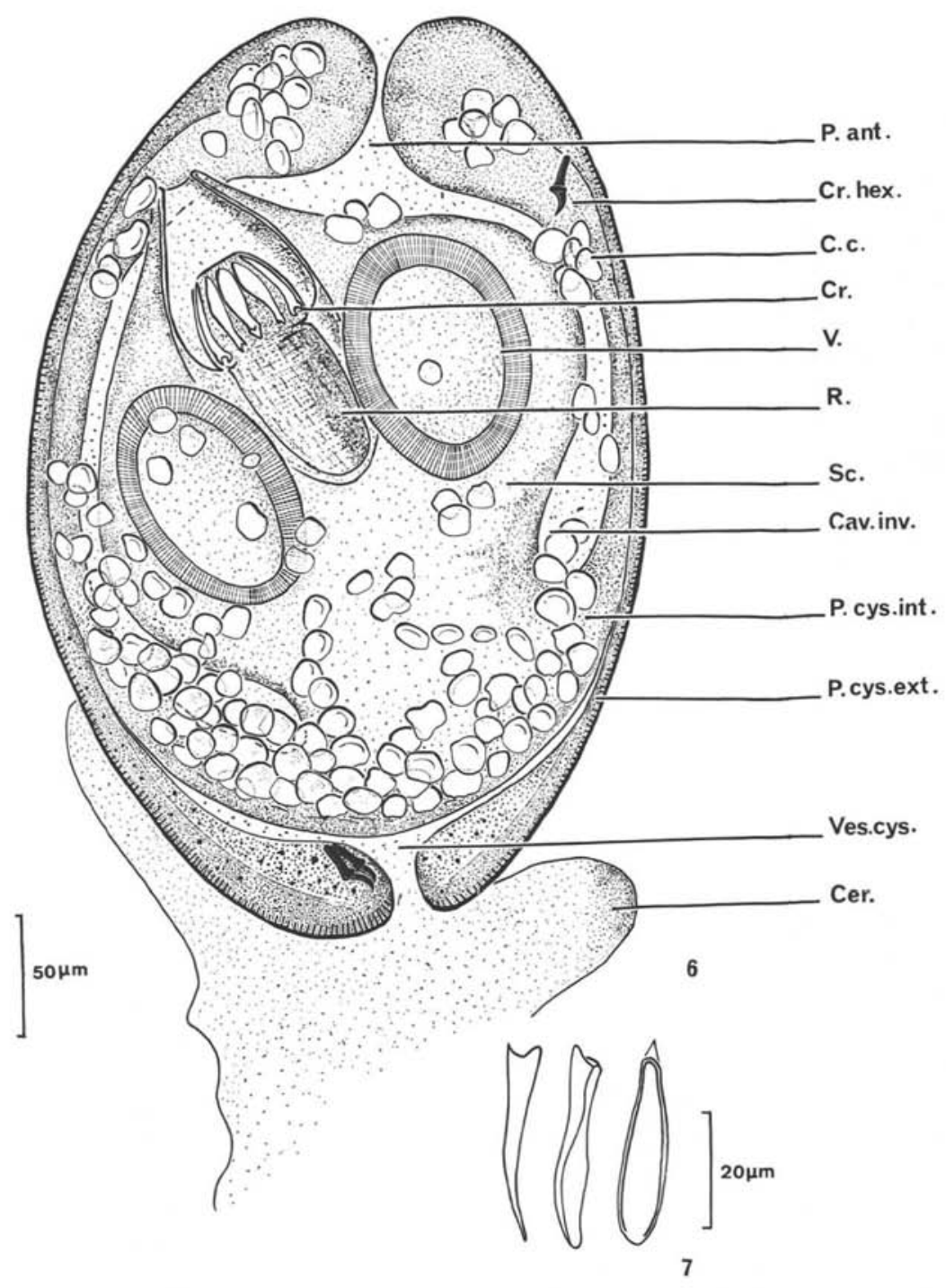

FIg. 6. - Structure du cysticercoïde à 26 jours. C.c. Corpuscule calcaire; Cav. inv. Cavité d'invagination; Cer. Cercomère; Cr. Crochet; Cr. hex. Crochet de la larve hexacanthe : P. cys. ext. Paroi cystique externe ; P. cys. int. Paroi cystique interne ; P. ant. Pore antérieur : R. Rostre ; Sc. Scolex ; V. Ventouse ; Vés. cys. Vésicule cystique.

Fig. 7. - Crochets du rostre.

Planche I. - H. stylosa. $a$. - Cysticercoïde à 5 jours. A la partie postérieure, on peut observer la présence des crochets de la larve hexacanthe (Cr.). Le cercomère (Cer.) apparaît. Vés. cys. Vésicule cystique. $b$. - Cysticercoïde à 7 jours. La division du cysticercoïde en trois parties est nette. Le scolex (Sc.) est muni d'un rostre (R.) ; les ventouses (V.) apparaissent. La paroi de la vésicule cystique (Vés. cyst.) s'épaissit. c. - Cysticercoïde à 8 jours. Le scolex bien différencié commence à s'invaginer. La vésicule cystique se déforme. d. - Cysticercoïde à 14 jours. Le scolex (Sc.) est invaginé ; le cysticercoïde est enveloppé par un glycocalyx (Gx) abondant. Le cercomère (Cer.) est long et boursouflé par endroit. 

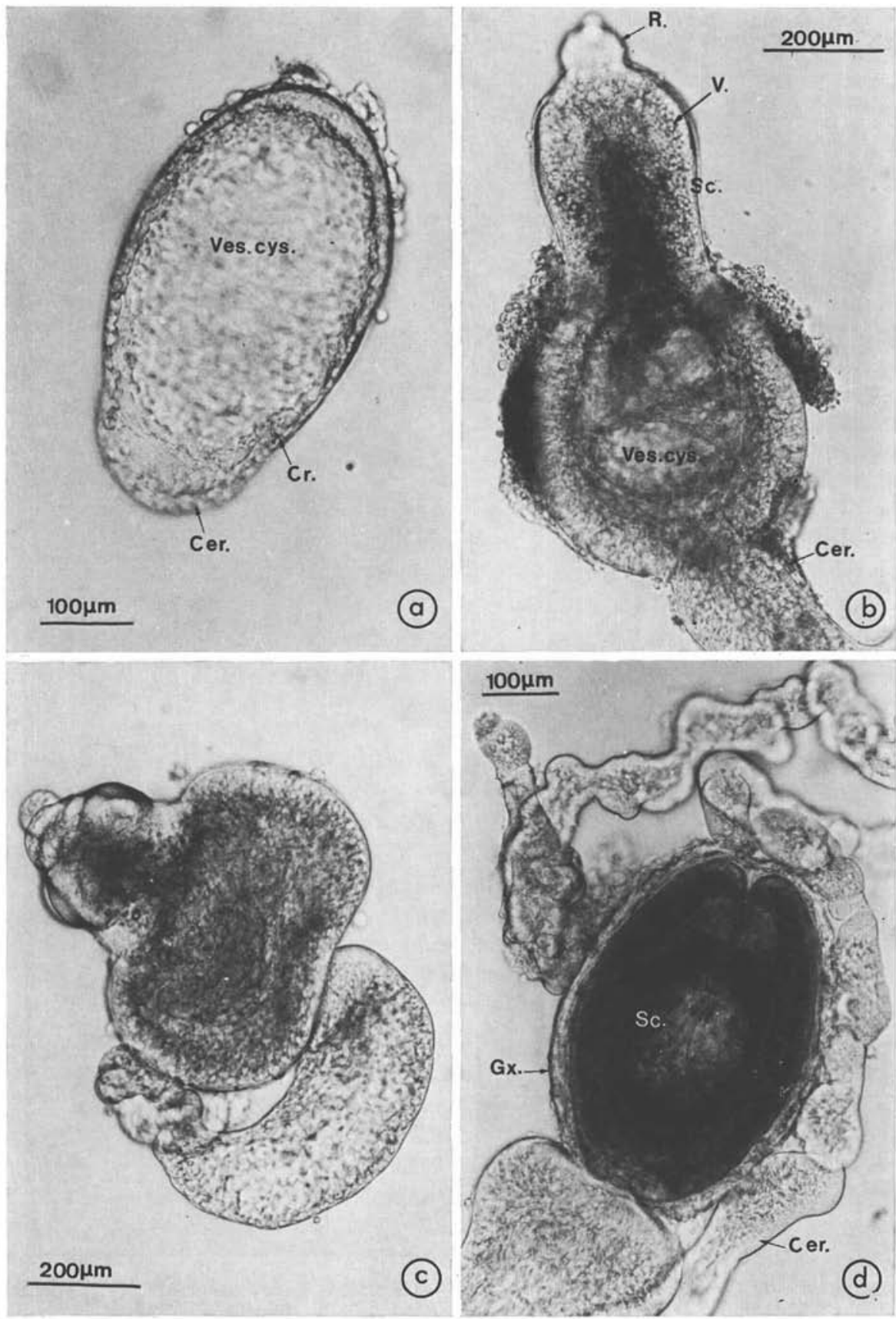

Planche I 


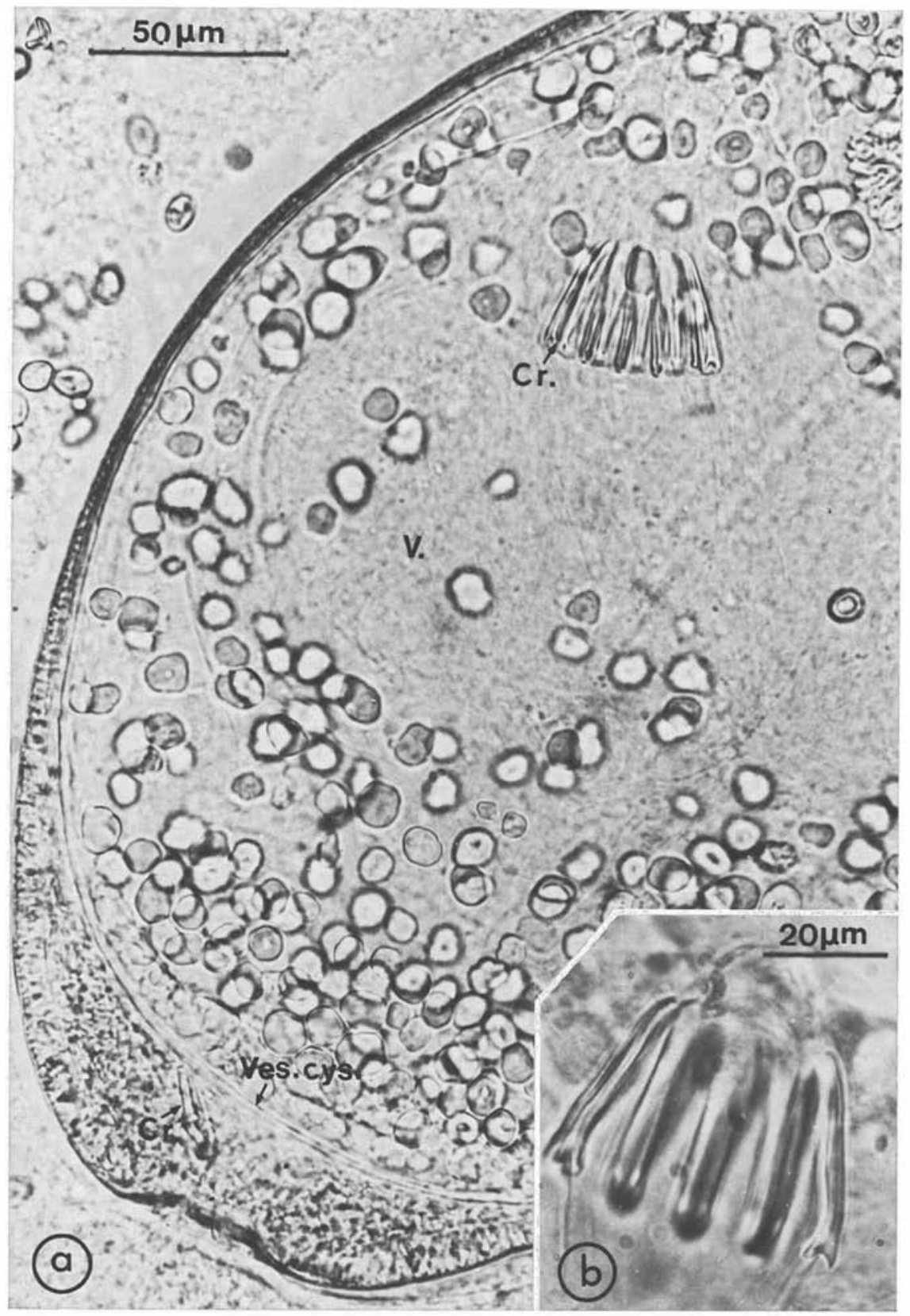

Planche II. - Cysticercoïde à 26 jours. a. - Structure du cysticercoïde. Cr. Crochets ; V. Ventouse; Vés. cys. Vésicule cystique. b. - Crochets du rostre. 
1958 ; Voge et Heyneman, 1958 ; Voge, 1961) pourrait être en relation avec l'aug. mentation de la température, les Orthoptères étant maintenus en élevage à $34^{\circ} \mathrm{C}$. D'autre part, chez L. migratoria, la morphologie du cercomère est différente. Il présente, par endroits, des vésicules qui simulent des vésicules cystiques à l'intérieur desquelles n'apparaît néanmoins aucune différenciation. Certaines de ces vésicules montrent des aspects de dégénérescence mélanique.

\section{Etude du développement chez Dermestes frischi.}

11 jours après l'infestation, la dissection des larves de Dermestes n'a pas révélé la présence de cysticercoïdes dans l'abdomen. Cependant, de nombreuses oncosphères ont été observées dans la muqueuse de l'intestin moyen. Il semble donc que l'éclosion des œufs d' $H$. stylosa ait bien lieu dans la lumière du tube digestif. Les oncosphères pénètrent dans l'épithélium intestinal, mais ne peuvent achever leur migration. La plupart, libérées de leurs enveloppes embryonnaires, sont représentées par des amas. de pigments mélaniques d'où émergent les crochets.

Le même phénomène de dégénérescence a été observé par Cavier et Léger (1965) pour les cysticercoïdes d'Hymenolepis nana var. fraterna introduits expérimentalement dans l'hémocèle de Periplaneta americana.

Ces larves hexacanthes en dégénérescence sont entourées de plusieurs couches de cellules fusiformes et par des macrocytes qui forment une capsule réactionnelle. $\mathrm{Ce}$ processus de dégénérescence s'apparente à celui observé chez Ctenocephalides felis parasitée par Dipylidium caninum (Chen, 1934).

L'impossibilité pour l'oncosphère d'H. stylosa de traverser la paroi intestinale po:urrait résulter, comme dans le cas des larves de $T$. molitor, infestées expérimentalement par $H$. diminuta (Voge et Graiwer, 1964), de l'importance de la membrane péritrophique ou de la densité de l'épithélium. Seule l'infestation de Dermestes adultes pourrait nous renseigner. Malheureusement, tous nos essais ont jusqu’à présent échoué.

\section{Discussion}

Baer (1973) écrivait, à propos du développement des Hymenolepididae : \& Un examen des formes larvaires montre peu de variations à partir du type cysticercoïde. Les appendices larvaires, qui sont très variables, sont des structures adaptatives en relation avec la nature de l'hôte intermédiaire. Elles possèdent surtout des fonctions trophiques et ne peuvent être d'une certaine aide du point de vue taxonomique. »

Le caractère «interne » (après invagination) ou « externe» (avant invagination) du développement du scolex des cysticercoïdes d'Hymenolepis de Mammifères prouve au contraire que les formes larvaires de ces parasites présentent d'importantes variations.

Le développement larvaire des Hymenolepis d'Oiseaux paraît plus homogène. Dans ce groupe, qu'il s'agisse de Cestodes d'Oiseaux aquatiques comme $H$. hopkinsi Schiller, 1951 (McLaughlin et Burt, 1970), ou H. furcifera Krabbe, 1869 (Jarecka,- 
1958), tous deux parasites d'Anatidés, ou de Cestodes d'Oiseaux terrestres comme H. exigua Yashida, 1908 (Alicata et Chang, 1939), parasite de Gallinacés, ou H. stylosa, parasite de Corvidés, l'évolution des formes larvaires est identique. Il s'agit de cysticercoïdes «externes» chez lesquels le scolex se différencie avant invagination dans la vésicule cystique et qui, au terme de leur évolution, sont pourvus d'un cercomère bien développé.

Baer pensait que la structure du cysticercoïde était en relation avec la nature de l'hôte intermédiaire. Le meilleur exemple est $H$. nana, chez lequel le cysticercoïde est différent selon que l'évolution se fait chez Tribolium confusum (Schiller, 1959) ou chcz la Souris (Hunninen, 1935; Caley, 1975). Cela ne semble pas être la règle pour les Hymenolepis d'Oiseaux. Il est remarquable de constater, en effet, que les formes larvaires de ces Cestodes ne présentent aucune différence morphologique et ont une évolution semblable dans leurs hôtes intermédiaires respectifs, qu'il s'agisse de Crustacés, de Mollusques ou d'Insectes, malgré les différences importantes que supposent ces types d'hôtes: $\mathrm{T}^{\circ}, \mathrm{pH}$, pression osmotique, nature des matières nutritives.

D'autre part, le cysticercoïde d'H. stylosa présente la même évolution et la même morphologie chez un Coléoptère, Tenebrio molitor, et chez un Orthoptère, Locusta migratoria. Un résultat identique a été obtenu chez $H$. farciminosa, dont le cysticercoïde se développe chez divers Orthoptères (Dutt et Méhra, 1962) et chez des Coléoptères, Pterostichus madidus (Mourad, 1967) et Amara convexinscula (Gabrion, non publié), sans que l'on puisse déceler de différences morphologiques entre les cysticercoïdes.

Il semble donc que, dans ce groupe d'Hymenolepis, l'évolution du cysticercoïde et la présence d'un cercomère bien développé constituent des caractères constants, d'une certaine valeur taxonomique.

\section{Conclusion}

Le cysticercoïde d'H. stylosa, par sa morphologie et son évolution, est semblable aux cysticercoïdes des autres Hymenolepis d'Oiseaux, dont le développement, naturel ou expérimental, est connu.

Une étude comparative, à l'aide de la microscopie électronique, des cysticercoïdes d'Hymenolepis d'Oiseaux et de Mammifères, s'impose. Une telle étude devrait permettre de préciser si la morphologie et l'évolution des cysticercoïdes peuvent nous renseigner sur la nature des affinités familiales des espèces appartenant à ce genre, ou si, comme le suggérait Baer (1957), « à l'évolution des vers adultes est venue se greffer, secondairement, une évolution des larves qui masque ainsi les rapports originels entre les hôtes définitifs et les hôtes intermédiaires, en introduisant un facteur écologique nouveau...». 


\section{Bibliographie}

Alicata (J. E.) et Chang (E.), 1939. - The life-history of Hymenolepis exigua a Cestode of poultry in Hawaii. J. Parasitol., 25, 121-127.

BAER (J.-G.), 1957. - Répartition et endémicité des Cestodes chez les Reptiles, Oiseaux et Mammifères. Symp. Spec. Parasit. Neuchâtel, 270-291.

BAER (J.-G.), 1973. - Considerations on the classifications of the family Hymenolepididae (Cestoda, Cyclophyllidea). Materials of the International conference of Hymenolepididae Warsawa, 147-153.

CALeY (J.), 1975. - A comparative study of the two alternative larval forms of Hymenolepis nana, the dwarf tapeworm with special reference to the process of exystment. $Z$. Parasitkde 47, 217-235.

CAVIER (R.) et LÉGer (N.), 1965. - A propos de l'évolution d'Hymenolepis nana var fraterna chez les hôtes intermédiaires inhabituels. Ann. Parasitol., hum. comp., 40, 651-658.

CHEN (H. T.), 1934. - Reactions of Ctenocephalides felis to Dipylidium caninum. Zeit. $f$. Parasit. 6, 603-637.

Dutt (S. C.) et Mehra (K. N.), 1962. - Studies on the life history of Hymenolepis farciminosa (Goeze, 1782) (Cestoda, Hymenolepididae). Parasitology, 52, 397-400.

Freeman (R.S.), $1952 a$. - The biology and life history of Monoecocestus Beddard, 1914 (Cestoda, Anoplocephalidae) from the porcupine. J. Parasitol., 35, 605-612.

Heyneman (D.), 1958. - Effect of temperature on rate of development and viability of the cestode Hymenolepis nana in its intermediate host. Exp. Parasitol., 7, 374-382.

HUNNINEN (A. V.), 1935. - Studies on the life history and host-parasite relations of Hymenolepis fraterna ( $H$. nana var fraterna Stiles) in white mice. Amer. J. Hyg., 22, 414-443.

JAFECKA (L.), 1958. - Cladocera as the intermediate hosts of certain species of Cestoda. Life cycle of Anomotaenia ciliata (Fuhr., 1913) and Hymenolepis furcifera (Krabbe, 1869). Bull. Acad. Pol. Sci., 6, 157-166.

MCLaughlin (J. D.) et Burt (M.), 1970. - Observations on the morphology and life cycle of Hymenolepis hopkinsi Schiller, 1951 (Cestoda, Cyclophyllidea), a parasite of black ducks (Anas rubripes Brewster). Can. J. Zool., 48, 1043-1046.

Mettrick (D. F.), 1958. - Helminth parasites of Hertfordshire birds. II. Cestoda. J. Helminthol., 32, 159-194.

Mourad (A. R.), 1967. - Beetles as intermediate hosts of Cestodes of the family Hymenolepididae. Folia Parasitol., 14, 379-380.

SCHILler (E. L.), 1959. - Experimental studies on morphological variation in the Cestode genus Hymenolepis. 1. Morphology and development of the cysticercoïd of $\mathrm{H}$. nana in Tribolium confusum. Exp. Parasitol., 8, 91-119.

VGGE (M.), 1961 - Effect of high temperature stress on histogenesis in the cysticercoïd of Hymenolepis diminuta (Cestoda; Cyclophyllidea). J. Parasitol., 47, 189-195. 
Voge (M.) et Graiwer (M.), 1964. - Development of Oncospheres of Hymenolepis diminuta, hatched in vivo and in vitro, in the larvae of Tenebrio molitor. J. Parasitol., 50, 267-270.

Voge (M.) et Heyneman (D.), 1957. - Development of Hymenolepis nana and Hymenolepis diminuta (Cestoda, Hymenolepididae) in the intermediate host 1 ribolium confusum. Univ. Calif., Berkeley, Publ. Zool., 59, 549-580.

Voge (M.) et Heyneman (D.), 1958. - Effect of high temperature on the larval development of Hymenolepis nana and Hymenolepis diminuta (Cestoda, Cyclophyllidea). J. Parasitol., 44, 249-260. 